\title{
Modelos de crecimiento diametral para Austrocedrus chilensis en la Cordillera de Nahuelbuta, Chile: Una interpretación biológica
}

\author{
Eduardo Navarrete Espinoza ${ }^{1,2}$, Juan Cárcamo Ojeda ${ }^{1}$ \\ y Pablo Novoa Barra ${ }^{1,2}$ \\ ${ }^{1}$ Departamento Forestal, Unidad Académica Los Angeles, Universidad de Concepción y \\ ${ }^{2}$ Grupo de Estudios Silvoecológicos (GESE). Juan Antonio Coloma 0201. Casilla 341. Los Angeles, Chile.
}

\begin{abstract}
E. Navarrete Espinoza, J. Cárcamo Ojeda, and P. Novoa Barra. 2008. Diameter growth models for Austrocedrus chilensis in the Nahuelbuta Mountain Range, Chile: A biological interpretation. Cien. Inv. Agr. 35(3):311-320. This study was performed in three stands of "ciprés de la cordillera" (Austrocedrus chilensis), at Horno de Barro, Lomas de Toro and Las Animas, in the Maitenrehue area $\left(37^{\circ} 30^{\prime}-39^{\circ} 37^{\prime} \mathrm{S} ; 7^{\circ} 45^{\prime}-73^{\circ} 00^{\prime} \mathrm{W}\right)$ located in the Nahuelbuta mountain range in the Araucanía Region. This area is considered a glacial refuge for many species of the Chilean forests. By means of individual tree analyses and competition assessments, diameter increment models were built to explain and estimate diameter growth based on dendrometric and dasometric variables. Data was gathered by stem analysis. The results presented refer to the diameter at chest level (1.3 m above soil level) (DAP) mean annual increment models for each sampled area, which involved a single predictive variable of girth breast height. Secondly, for the Maitenrehue area, a general model of DAP mean annual increments depended on the distance, and it involved variables related to the trees and competitors. Statistical differences amongst linear models for all areas analyzed reveals that A. chilensis is growing in diverse settings and with different growth patterns in response to intraspecific and interspecific competition faced by this species.
\end{abstract}

Key words: Andean cypress, individual growth, specific competition.

\section{Introducción}

El último censo forestal realizado en 1999, entregó una valiosa información acerca del estado de los bosques en Chile. De acuerdo con estos datos, el ciprés de la cordillera (Austrocedrus chilensis (D. Don) Florin et Boutelje) es la especie de importancia forestal más escasa, con sólo el $0,3 \%$ de un total de 13.443.316 ha de bosque nativo (CONAFCONAMA, 1999). Actualmente en Chile, A. chilensis se encuentra a lo largo de la Cordillera de Los Andes desde la V Región (32 $39^{\circ}$ S) hasta la cuenca del río Palena, X Región $\left(44^{\circ} \mathrm{S}\right)$, en condiciones ambientales muy rigurosa que impiden un buen desarrollo (Donoso, 1993),

Recibido 22 enero 2008. Aceptado 30 junio 2008.

${ }^{1}$ Dirigir correpondencia a E. Navarrete: ednavarr@udec.cl
Los bosques de A. chilensis se encuentran especialmente en la vertiente oriental de la Cordillera de Los Andes, donde alcanzan un mejor desarrollo, extendiéndose desde los $38^{\circ} 30^{\prime}$ S hasta los 4335' S (Tortorelli, 1956). En la Cordillera de la Costa se encuentra escasamente entre $37^{\circ} 30^{\prime} \mathrm{S}$ y $39^{\circ} 37^{\prime} \mathrm{S}$, en pendientes rocosas y a orillas de cursos de agua. Austrocedrus chilensis presenta poblaciones fragmentadas en la Cordillera de Nahuelbuta $\left(38^{\circ} \mathrm{S}\right.$, aproximadamente, entre 1.000 y 1.500 m.s.n.m.) (Veblen et al., 1995).

Los efectos negativos de la creciente presión humana sobre los bosques en Chile, se han extendido a este tipo de ecosistemas. Existen diferentes estudios que indican que los factores exógenos inciden en la dinámica estructural de un bosque, como son las limitantes ambientales y régimen de disturbios; o endógenos, como las 
características de la especie y las interacciones entre individuos. Los bosques de A. chilensis presentan diferentes patrones de regeneración y desarrollo poblacional en condiciones variables de disponibilidad de agua en el suelo. Las condiciones iniciales de densidad y distribución espacial pueden afectar las condiciones de todo el desarrollo estructural, ya que determinan la intensidad de la competencia entre individuos (Veblen et al., 1995). Estudios comparativos sobre rodales chilenos, indican que A. chilensis es la conífera con más rápido crecimiento en Chile (Donoso, 1981).

El crecimiento de un organismo se puede describir a través de modelos que engloban variables endógenas y exógenas. El análisis de los patrones de crecimiento individual, a través del estudio de anillos de crecimiento, es una fuente de información importante para conocer los cambios en los crecimientos individuales, especialmente de los árboles del estrato superior (dominantes y codominantes), los cuales tienen efectos importantes sobre la culminación de los crecimientos y rendimientos del rodal y/o a nivel de árbol individual (Goya et al., 1998). Debido a la sensibilidad al micrositio de la especie a estudiar, y a las variaciones climáticas de Chile, mientras más acotada sea la población de estudio en cuanto a clima y suelo, mejores deberían ser las estimaciones, ya que podrían mejorar las variables de entrada (competencia), provocando los efectos que se quieren obtener en la variable de salida (crecimiento diametral) (Gutiérrez, 2000).

El siguiente estudio tuvo como objetivo modelar el crecimiento diametral de $A$. chilensis en la Cordillera de la Costa, para lo cual se formularon modelos de incremento medio basados en variables dendrométricas y dasométricas.

\section{Materiales y métodos}

\section{Area de estudio}

El área de estudio se realizó en la Cordillera de Nahuelbuta (IX Región, Araucanía, 38 S) de la Cordillera de la Costa, en un área donde actualmente $A$. chilensis participa en comunidad vegetal existente, considerada como refugio glacial para especies del bosque chileno (Villagrán, 2001). El sector denominado

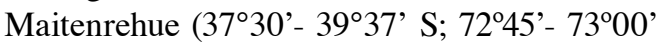
O) en la Cordillera de la Costa, presenta altitudes variables entre los 75 y los 1.195 m.s.n.m. Esta área se caracteriza por lomajes suaves y mesetas, suelos volcánicos, con presencia de suelos rojoarcillosos hasta los 400 m.s.n.m. (Veit y Garleff, 1995). Presenta clima templado lluvioso con influencia oceánica, vientos predominantes del oeste e influencia polar. La precipitación anual es cercana a $3.000 \mathrm{~mm}$ (Armesto, 1997; Di Castri y Hajek, 1976). La vegetación del sector se caracteriza por la presencia de A. chilensis, además de, Nothofagus obliqua (Mirbel.) Oerst, Cryptocarya alba (Mol.) Looser, Lomatia hirsuta (Lam.) Diels ex Macbr, Lomatia dentata (R.et P.) R. Br., Lithrea caustica (Mol.) H. et A., Persea lingue Nees, Luma apiculata (DC.). Burret, Podocarpus saligna D. Don y Peumus boldus Mol.

\section{Selección de los árboles sujetos y competidores}

Se seleccionaron tres sectores de estudio, elegidos a escala de paisaje, los que se denominaron: Horno de Barro ( $37^{\circ} 38^{\prime}$ S; $72^{\circ} 52^{\prime}$ O), Lomas de Toro ( $37^{\circ} 37^{\prime} \mathrm{S}$; $72^{\circ} 50^{\prime}$ O) y Las Animas ( $37^{\circ} 37^{\prime} \mathrm{S} ; 7^{\circ} 49^{\prime} \mathrm{O}$ ), los que se ubican principalmente a orillas de cursos de agua. Se establecieron ocho unidades muestrales temporales de $900 \mathrm{~m}^{2}$ en los sectores en estudio. En cada unidad se seleccionaron los árboles muestra (árboles sujeto) y árboles competidores, los que correspondieron a los árboles más cercanos a los árboles sujeto (distancia $<5 \mathrm{~m}$ ), independiente de la especie. Tanto a los árboles sujeto, como a los árboles competidores se caracterizaron respecto del diámetro a $1,3 \mathrm{~m}$ desde el suelo (DAP), diámetro a 0,3 $\mathrm{m}$ desde el suelo (DAT), altura total (HT), altura comienzo de copa viva (HCC) y diámetros de copa a través de proyección vertical $(\mathrm{N}-\mathrm{S} ; \mathrm{E}-\mathrm{O})$ de cada uno de los individuos con un DAP $>4 \mathrm{~cm}$. Además, en cada unidad muestral se registró la exposición y pendiente.

\section{Obtención de la información básica}

Con la ayuda de un taladro epidométrico se obtuvo un tarugo desde cada árbol sujeto, tanto 
al DAP, como al DAT. El crecimiento histórico de los individuos, se determinó midiendo el espesor de los anillos de crecimiento con un pie metro digital a la altura del DAP. La edad se determinó por medio del conteo de anillos en los tarugos a la altura del DAT.

\section{Modelos de crecimiento diametral}

El incremento medio anual (IMA) en diámetro se determinó calculando el promedio aritmético de los incrementos para cada tarugo obtenido a la altura del DAP. Esta información permitió establecer una relación entre el IMA en diámetro, y las variables de estado del árbol sujeto y sus respectivos árboles competidores, las cuales se procedió a dividirlas en cuatro diferentes grupos (Trincado y Real, 2002): 1. Variables de tamaño de árboles sujeto, correspondiente a variables de estado que representan la dimensión del individuo (área basal, área de copa (asumiéndola circular), diámetro de copa, DAP, DAT, HT, HCC). 2. Variables de vigor, correspondiente a la capacidad fotosintética del individuo, representado por la razón de copas $(\mathrm{RC})(\mathrm{RC}=[(\mathrm{HT}-\mathrm{HCC}) / \mathrm{HT}] \mathrm{x} 100) \mathrm{y}$ el coeficiente de esbeltez (COEF ESB=HT/ DAP). 3. Variables de sitio, correspondiente a la transformación propuesta por Stage (1976). Esta transformación incorporó las variables de exposición (EXP) y pendiente (PEND), medidas en grados, como un efecto circular continuo, cancelando el efecto de exposición cuando la pendiente fue cero [SITIO = PEND + PEND x COS(EXP) + PEND x SEN(EXP)] 4. Variables de competencia, correspondiente al índice de competencia a nivel de árbol individual de Glover y Hool, el cual cuantifica el grado de competencia que afecta a un árbol $\left(\right.$ COMPETEN $\left.=d_{i}^{2} / \overline{D^{2}}\right)$, donde $d_{i}=$ diámetro (cm) del árbol sujeto y $\bar{D}=$ diámetro $(\mathrm{cm})$ promedio del rodal (Martínez, 2005).

\section{Análisis estadísticos}

Con las variables registradas se procedió a ajustar un modelo lineal general para el IMA, de la forma:

$$
Y_{i}=\beta_{0}+\sum_{i=1}^{k} \beta_{k} X_{i}+\varepsilon_{i}
$$

Donde $Y_{i}=$ vector $\mathrm{N}_{\mathrm{i}} \times 1$ de observaciones para el i-ésimo árbol, $\beta=$ vector $\mathrm{P} \times 1$ de coeficientes de regresión, $\mathrm{P}=\mathrm{k}+1, X_{i}=$ matriz no estocástica $\mathrm{N}_{\mathrm{i}} \times \mathrm{P}$ de rango $\mathrm{P}$ de observaciones en las variables predictoras y $\varepsilon_{i}=$ vector $\mathrm{N}_{\mathrm{i}} \times 1$ de errores aleatorios.

La selección de las variables independientes a ingresar en los modelos, se realizó por regresión paso a paso (forward stepwise regression y backward stepwise regression), usando Statistica 5.5 (StatSoft Inc., Tulsa, OK, EUA). A la vez se realizó el análisis de los supuestos involucrados en la construcción de modelos lineales, correspondientes a normalidad, homocedasticidad e independencia de los residuos (Neter et al., 1996).

Las variables independientes analizadas para la formulación del modelo en función de variables predictoras provenientes de los árboles sujetos y de los árboles competidores fueron: DAP = diámetro altura del pecho (a 1,3 m de altura, en $\mathrm{cm})$, DAT = diámetro altura del tocón (a $0,3 \mathrm{~m}$ de altura, en $\mathrm{cm}), \mathrm{HT}=$ altura total $(\mathrm{en} \mathrm{m})$, $\mathrm{HCC}=$ altura comienzo de copa viva (en $\mathrm{m}$ ), DIAM COP $=$ Diámetro promedio de copa (en $\mathrm{m})$, AREA COP = área de copa $\left(\mathrm{en}^{2}\right)^{2}, \mathrm{COEF}$ $\mathrm{ESB}=$ coeficiente de esbeltez, RAZON COP = razón de copas, COMPETEN $=$ índice de competencia individual (Glover y Hool), SITIO = valor de la función propuesta por Stage (1976), DIST_COM = distancia al árbol competidor (m), DAP_C = diámetro altura del pecho (a 1,3 $\mathrm{m})$ del competidor $(\mathrm{cm})$, DAT_C $=$ diámetro altura del tocón (a $0,3 \mathrm{~m}$ ) del competidor $(\mathrm{cm})$, HT_C= altura total del competidor (m), HCC_ $\mathrm{C}=$ altura comienzo de copa del competidor $(\mathrm{m})$, DIAM COP_C $=$ diámetro promedio de copa del competidor (m), AREA COP_C = área de copa del competidor $\left(\mathrm{m}^{2}\right)$, COEF ESB_C = coeficiente de esbeltez del competidor, RAZON COP_C $=$ razón de copas del competidor $\mathrm{y}$ COMPETEN_C = índice de competencia individual (Glover y Hool) del competidor.

Posteriormente, se agruparon las variables independientes por cada sector muestreado, de modo de construir un modelo de incremento medio anual en DAP para cada uno de ellos.

Para identificar las diferencias que pudieran 
existir entre los modelos de crecimiento por sector, se utilizó la prueba de Chow, aplicando la prueba $\mathrm{F}$ de Fisher, que detecta posibles diferencias entre modelos (Gujarati, 1992); y la prueba $t$ de Student, para determinar si la diferencia se debe a uno o más parámetros, simultáneamente (Mora et al., 1996). Los estadísticos de prueba $\mathrm{F}$ y $\mathrm{t}$ fueron:

Prueba de Chow:

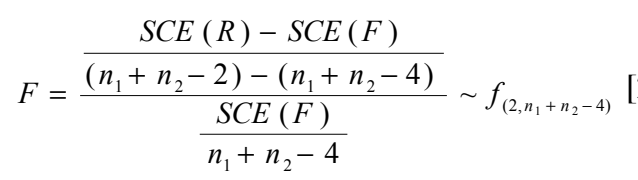

donde, SCE (R) = Suma de cuadrados del error del modelo conjunto entre dos sectores a comparar. SCE $(F)$ = Adición de suma de cuadrados del error de los modelos de sectores a comparar. $n_{1+} n_{2}-2=$ Grados de libertad de SCE (R). $n_{1+} n_{2}-4=$ Grados de libertad de SCE (F).

\section{Prueba t:}

$$
T=\frac{\hat{\beta}_{s i}-\hat{\beta}_{s j}}{\sqrt{\operatorname{vâr}\left(\hat{\beta}_{s i}-\hat{\beta}_{s j}\right)}} \sim t_{\left(n_{s i}-p-1+n_{s j}-p-1\right)}
$$

donde, $\beta_{s i}=$ Parámetros del modelo para el sector i-ésimo, $\hat{\beta}_{s j}=$ Parámetros del modelo para el sector j-ésimo, vâr $\left(\hat{\beta}_{s i}-\hat{\beta}_{s j}\right)=$ Suma de las varianzas de los parámetros del modelo y $p=$ Número de parámetros

Finalmente, y con la totalidad de la información de las variables registradas para cada sector, se construyó un modelo general de incremento medio anual para DAP, donde se analizaron las variables dendrométricas y dasométricas anteriormente mencionadas, junto con las variables de sitio, exposición y pendiente.

\section{Resultados y discusión}

\section{Situación actual de los rodales estudiados}

El sector Horno de Barro presentó el menor número de árboles por hectárea, junto con el mayor DAP promedio y; por ende, mayor área basal, lo que nos indica que A. chilensis, al estar sometido a una menor competencia intraespecífica, logra un mayor desarrollo (Cuadro 1). En Horno de Barro (Figura 1a), A. chilensis presentó una estructura diamétrica bimodal, con un bajo reclutamiento y dos estratos bien diferenciados. Se apreció una baja alteración antrópica, existiendo la mayoría de las clases de diamétricas, esto debido a que fue el sector de menor presión de uso generada por el tránsito humano y animal (Rubilar, 2002).

Lomas de Toro fue el sector que presentó el mayor número de árboles por hectárea y a su vez, los menores valores de DAP promedio, área basal y altura media, indicando la existencia de individuos jóvenes y una mayor competencia intraespecífica (Cuadro 1). El sector Lomas de Toro (Figura 1b) presentó un reclutamiento de la especie mayor que el sector Horno de Barro, con una alta presencia de individuos en las clases inferiores, existiendo una estructura descrita por Baker (1950), como irregular. Se evidencia la ausencia de clases diamétricas intermedias, debido principalmente a actividades antrópicas predominantes en el sector.

En el sector Las Animas (Figura 1c) existió un bajo reclutamiento de la especie, representado por una estructura diamétrica regular.

\section{Caracterización de las variables de estado}

En el Cuadro 2 se observa que las variables de tamaño correspondientes al árbol sujeto (DAP, DAT, HT, HCC, área de copa y diámetro de copa), presentaron características diferentes en cada sector muestreado, las cuales, no son atribuibles a sus respectivas edades. Esto nos indicaría que los sectores en que se está desarrollando A. chilensis, presentan características de sitio diferentes.

Los sectores donde A. chilensis presenta la menor competencia son Horno de Barro y Las Animas; en donde se observaron mayores valores para el índice de competencia de Glover y Hool (COMPETEN), mayores diámetros y áreas de copa; y por consiguiente, donde se registró un mayor incremento medio anual promedio, con $0,4 \mathrm{~cm}$ y $0,3 \mathrm{~cm}$, respectivamente (Cuadro 2). La altura de la 
Cuadro 1. Sectores de Austrocedrus chilensis y promedios de variables consideradas en este estudio.

Table 1. Sectors of the Austrocedrus chilensis forest and mean of the variables analyzed in these studies.

\begin{tabular}{lrrr}
\hline Variables & \multicolumn{3}{c}{ Sectores } \\
\cline { 2 - 4 } & $\begin{array}{c}\text { Horno } \\
\text { de Barro }\end{array}$ & $\begin{array}{c}\text { Lomas } \\
\text { de Toro }\end{array}$ & $\begin{array}{c}\text { Las } \\
\text { Animas }\end{array}$ \\
\hline${\text { Arboles, } \mathrm{n}^{\circ} \cdot \mathrm{ha}^{-1}}^{133,0}$ & 190,0 & 186,0 \\
DAP $^{1}, \mathrm{~cm}$ & 19,1 & 9,4 & 14,9 \\
Area basal, $\mathrm{m}^{2} \cdot \mathrm{ha}^{-2}$ & 44,7 & 4,1 & 34,1 \\
Altura media, $\mathrm{m}$ & 10,6 & 6,8 & 10,7 \\
\hline
\end{tabular}

${ }^{1} \mathrm{DAP}=$ diámetro a la altura del pecho (a 1,3 $\mathrm{m}$ de altura).

especie, es también un parámetro que puede seguir este patrón de comportamiento frente a la competencia expuesta, donde árboles que crecieron en condiciones de alta competencia, alcanzaron y mantuvieron por largo tiempo tasas de crecimiento inferiores a otras plantas que se desarrollan más libremente (Ferrando $e t$ al., 2001). Esto queda de manifiesto en el sector Lomas de Toro, donde A. chilensis alcanza la mayor competencia, y en consecuencia, una menor altura promedio.

Cabe señalar, que en los sectores Horno de Barro y Las Animas, la asociación más frecuente de $A$. chilensis fue con N. obliqua, concordando con lo descrito por Donoso (1993), quien menciona que el mejor desarrollo de $A$. chilensis se obtiene al asociarse con robles en las exposiciones sur y cerca de fondos de quebradas. Esta situación que se presentó en estos dos sectores, donde los robles alcanzaron el estrato dominante. En contraposición, el sector Lomas de Toro, A. chilensis presentó una mayor densidad, lo que generó una mayor competencia intraespecífica, junto con la existencia de suelos más pobres y delgados, limitando su crecimiento y en consecuencia, registrando el menor incremento diametral $(0,2 \mathrm{~cm})$. Lo anterior concuerda con lo descrito por Goya et al. (1998), quien menciona que $A$. chilensis presenta un menor incremento cuando se encuentra creciendo bajo el dosel de la misma especie.

Los bajos coeficientes de variación obtenidos para las variables edad y altura total en el sector Las Animas, (20,2 y 28,2\%, respectivamente) (Cuadro 2), indican una cierta coetaneidad del rodal, concordando con lo expuesto en el gráfico de distribución diamétrica del sector (Figura 1c), donde se observa un comportamiento estructural tendiente a la normalidad.
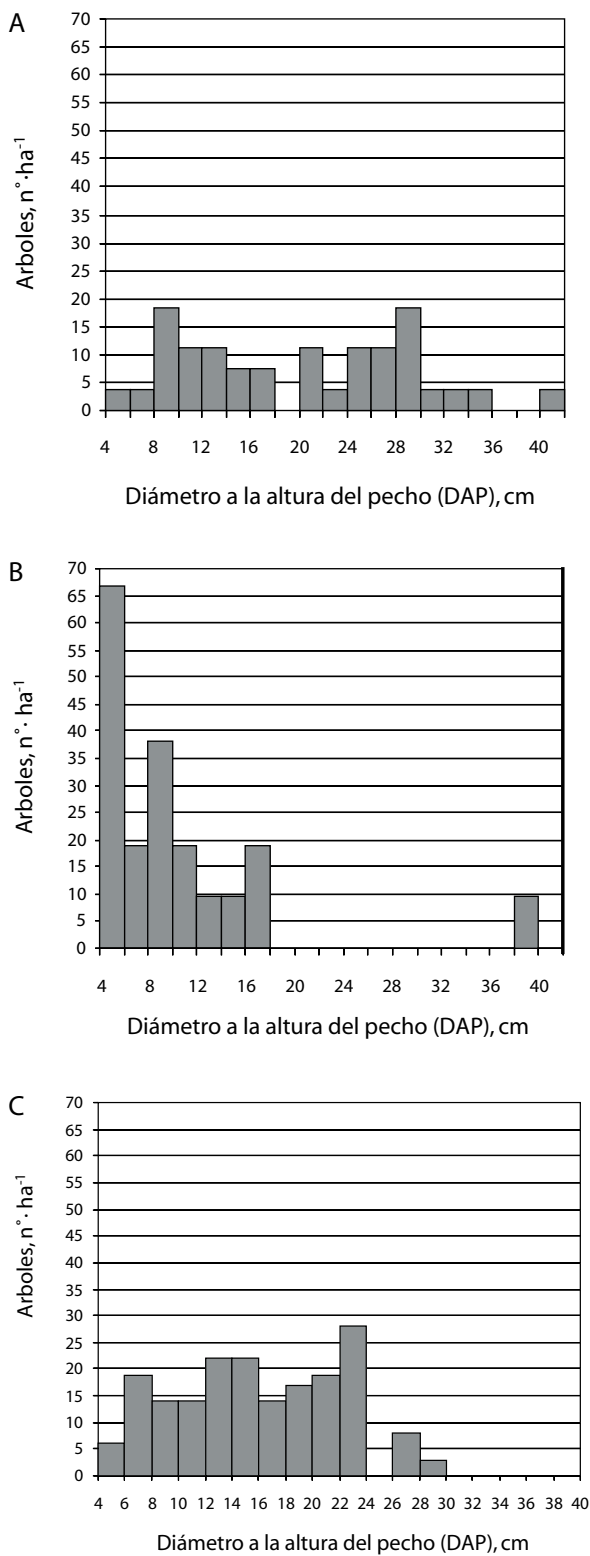

Figura 1. Distribuciones diamétricas de Austrocedrus chilensis en los sectores muestrados, Horno de Barro (A), Lomas de Toro (B) y Las Animas (C).

Figure 1. Diametric distributions of Austrocedrus chilensis in the sampled areas, Horno de Barro (A), Lomas de Toro (B), and Las Animas (C). 
Cuadro 2. Valores medios y coeficientes de variación de Austrocedrus chilensis obtenidas para las variables de estado de los árboles sujetos y árboles competidores.

Table 2. Mean values and variation coefficients obtained for Austrocedrus chilensis for the stated variables for subject trees and competitor trees.

\begin{tabular}{|c|c|c|c|c|c|c|}
\hline \multirow{3}{*}{ Variables de estado } & \multicolumn{6}{|c|}{ Sectores ${ }^{1}$} \\
\hline & \multicolumn{2}{|c|}{ Horno de Barro } & \multicolumn{2}{|c|}{ Lomas de Toro } & \multicolumn{2}{|c|}{ Las Animas } \\
\hline & Media & $\begin{array}{c}\mathrm{CV} \\
\%\end{array}$ & Media & $\begin{array}{c}\mathrm{CV} \\
\%\end{array}$ & Media & $\begin{array}{c}\mathrm{CV} \\
\%\end{array}$ \\
\hline Incremento medio anual en diámetro (IMA), $\mathrm{cm}$ & 0,4 & 35,2 & 0,2 & 50,4 & 0,3 & 37,8 \\
\hline Edad, años & 43,0 & 27,2 & 40,0 & 24,4 & 48,0 & 20,2 \\
\hline Diámetro altura del pecho (DAP), $\mathrm{cm}$ & 19,1 & 48,9 & 9,8 & 81,6 & 15,4 & 42,3 \\
\hline Diámetro altura del tocón (DAT), cm & 21,9 & 46,0 & 11,6 & 75,7 & 18,0 & 42,6 \\
\hline Altura total $(\mathrm{HT}), \mathrm{m}$ & 10,6 & 35,5 & 6,9 & 44,7 & 10,7 & 28,2 \\
\hline Altura cominezo copa viva (HCC), m & 2,2 & 47,3 & 1,3 & 54,5 & 3,7 & 43,9 \\
\hline Diámetro promedio de copa (DIAM COP), $\mathrm{m}$ & 4,3 & 45,2 & 2,5 & 55,1 & 3,9 & 33,7 \\
\hline Area de copa (AREA COP), $\mathrm{m}^{2}$ & 71,0 & 82,4 & 25,8 & 128,1 & 52,3 & 62,6 \\
\hline Coeficiente de esbeltez (COEF ESB) & 0,6 & 25,8 & 0,8 & 23,2 & 0,8 & 24,0 \\
\hline Razón de copas (RAZÓN COP) & 78,6 & 9,2 & 79,6 & 14,8 & 65,1 & 22,4 \\
\hline Indice de competencia individual (COMPETEN) ${ }^{1}$ & 1,2 & 85,8 & 0,4 & 209,5 & 0,7 & 83,8 \\
\hline Valor de la función propuesta (SITIO) ${ }^{1}$ & $-2,3$ & $-275,3$ & $-12,4$ & 0,0 & 5,9 & 52,5 \\
\hline Distancia al árbol competidor (DIST_COM), m & 2,6 & 69,4 & 0,7 & 56,1 & 1,4 & 73,1 \\
\hline Diámetro altura pecho del árbol competidor (DAP_C), cm & 18,2 & 55,8 & 8,7 & 48,7 & 18,7 & 208,4 \\
\hline Diámetro altura del tocón del árbol competidor (DAT_C), cm & 20,8 & 54,0 & 11,0 & 63,3 & 16,4 & 42,6 \\
\hline Altura total del árbol competidor (HT_C), m & 10,3 & 49,4 & 7,6 & 61,6 & 10,6 & 30,4 \\
\hline Altura comienzo de copa del árbol competidor (HCC_C), m & 2,5 & 92,9 & 1,4 & 52,6 & 3,9 & 47,4 \\
\hline Diámetro promedio de copa del árbol competidor (DIAM COP_C), m & 4,6 & 52,4 & 2,4 & 39,7 & 3,5 & 34,7 \\
\hline Area de copa del árbol competidor (AREA COP_C), $\mathrm{m}^{2}$ & 84,8 & 93,3 & 20,3 & 82,2 & 44,0 & 65,7 \\
\hline Coeficiente de esbeltez del árbol competidor (COEF ESB_C) & 0,6 & 34,4 & 0,9 & 27,0 & 0,8 & 28,4 \\
\hline Razón de copas del árbo competidor (RAZÓN COP_C) & 80,1 & 11,0 & 78,3 & 20,2 & 62,2 & 26,0 \\
\hline $\begin{array}{l}\text { Índice de competencia individual del árbol competidor } \\
(\text { COMPETEN_C })^{1}\end{array}$ & 1,0 & 56,0 & 1,0 & 49,0 & 1,0 & 207,9 \\
\hline
\end{tabular}

${ }^{1}$ COMPETEN COMPETEN C determinada según Glover y Hool. SITIO según Stage (1976).

\section{Construcción de los modelos de crecimiento por sector muestreado}

En el Cuadro 3 se presentan los modelos de IMA en DAP para cada sector muestreado.

Los coeficientes de correlación obtenidos en las tres ecuaciones fueron altamente significativos, destacando el modelo para el sector Lomas de Toro $(r=0,97)$, el cual presentó además, el menor error estándar de estimación y error cuadrático medio $(0,03 \mathrm{~cm} \mathrm{y} 14,64 \%$, respectivamente). Estos resultados de bondad de ajuste, se deben a que la mayor cantidad de individuos se concentró sólo en unas pocas clases de diámetro (Figura 1b), en comparación con los otros dos sectores muestreados, donde Horno de Barro y Las Animas (Figura 1a, 1c) evidenciaron una mayor variabilidad diametral.
De acuerdo con los resultados obtenidos por la prueba de Chow (Cuadro 4), aplicada a los modelos de cada sector, se determinó que el modelo ajustado al sector Horno de Barro presentó diferencias significativas respecto a los modelos de Lomas de Toro y Las Animas $\left(\mathrm{F}_{\mathrm{m}}>\mathrm{F}_{\mathrm{c}}\right)$. Esto sugiere que el crecimiento en Horno de Barro presentó características diferentes respecto a los otros sectores. A su vez, el estadístico de prueba, no detectó diferencias entre los modelos de Lomas de Toro y Las Animas; indicando que en estos dos sectores, no son estadísticamente distintos en las condiciones de crecimiento presentada por la especie. Sin embargo, la prueba de Chow no señaló explícitamente cuál de los coeficientes, intercepto o pendiente, fueron diferentes entre modelos. Por consiguiente, para identificar la fuente de dicha diferencia, se realizó una prueba t (Cuadro 5), indicando diferencias entre los 
Cuadro 3. Modelos lineales según el incremento medio anual en diámetro de Austrocedrus chilensis.

Table 3. Linear models based on the mean annual increase in diameter of Austrocedrus chilensis.

\begin{tabular}{lccccc}
\hline Sector & \multicolumn{1}{c}{ Modelo $^{1}$} & $\mathrm{r}$ & $\mathrm{R}^{2}$ & $\begin{array}{c}\mathrm{ECM}^{1} \\
\%\end{array}$ & $\begin{array}{c}\mathrm{EEE}^{1} \\
\mathrm{~cm}\end{array}$ \\
\hline $\begin{array}{l}\text { Horno de Barro } \\
(\mathrm{n}=36)\end{array}$ & $\mathrm{IMA}=0,161+0,013 \times \mathrm{DAP}$ & & & & $0,07 *$ \\
$\begin{array}{l}\text { Lomas de Toro } \\
(\mathrm{n}=20)\end{array}$ & $\mathrm{IMA}=0,097+0,012 \times \mathrm{DAP}$ & 0,72 & 19,76 & 0,03 \\
$\begin{array}{l}\text { Las Animas } \\
(\mathrm{n}=65)\end{array}$ & $\mathrm{IMA}=0,098+0,014 \times \mathrm{DAP}$ & $0,97^{*}$ & 0,94 & 14,64 & 0,04 \\
\hline
\end{tabular}

${ }^{1} \mathrm{IMA}=$ Incremento medio anual, $\mathrm{ECM}$ = Error cuadrático medio, EEE = Error estándar de estimación.

*, Altamente significativos $(\mathrm{p}=0,01)$.

interceptos del modelo de Horno de Barro con respecto a Lomas de Toro y Las Animas $\left(\mathrm{t}_{\mathrm{m}}>\mathrm{t}_{\mathrm{c}}\right)$, lo que significa que el IMA presentado por $A$. chilensis al inicio de su crecimiento en el sector Horno de Barro, fue mayor al registrado en los otros dos sectores (Figura 2). No se detectaron diferencias entre los interceptos de los modelos pertenecientes a Lomas de Toro y Las Animas, lo cual indica que la tasa crecimiento para IMA fue homogénea durante los primeros períodos de desarrollo de la especie en estos dos sectores (Figura 2). Esto se debería a que A. chilensis se habría iniciado en similares condiciones de cobertura arbórea; diferenciándose cada rodal, por la densidad inicial presentada por $A$. chilensis.

Las diferencias entre las pendientes de los modelos, nos indican que $A$. chilensis presenta diferentes tasas de crecimiento en cada uno de los sectores de muestreo, presentando el sector Horno de Barro, diferencias significativas con los otros dos sectores (Cuadro 5, Figura 2). Cabe señalar, que el estadístico $\mathrm{t}$, detectó también diferencias entre las pendientes de los modelos de Lomas de Toro y Las Animas (Cuadro 5). Esto nos indicaría, que el ritmo de crecimiento diametral presentado por la especie, también fue diferente entre estos sectores (Figura 2). Al inicio en el sector Las Animas A. chilensis tuvo un crecimiento similar al obtenido en Lomas de Toro, lo que se refleja por la similitud de sus interceptos (Cuadro 3 y Figura 2). A pesar de esto, Las Animas fue el sector en el cual A. chilensis presentó una mayor tasa de crecimiento $\left(\beta_{l}=0,014\right)$, tendiendo incluso a igualar al obtenido en el sector Horno de Barro, sobrepasando $42 \mathrm{~cm}$ de DAP (Figura
Cuadro 4. Resultados del análisis estadístico para la comparación entre modelos.

Table 4. Statistical comparison between models.

\begin{tabular}{|c|c|c|c|c|}
\hline \multirow[t]{3}{*}{ Sectores } & \multicolumn{4}{|c|}{ Sectores } \\
\hline & \multicolumn{2}{|c|}{ Horno de Barro } & \multicolumn{2}{|c|}{ Lomas de Toro } \\
\hline & $\mathrm{F}_{\mathrm{m}}$ & $\mathrm{F}_{\mathrm{c}}$ & $\mathrm{F}_{\mathrm{m}}$ & $\mathrm{F}_{\mathrm{c}}$ \\
\hline Lomas de Toro & 5,56 & 3,18 & - & - \\
\hline Las Animas & 4,34 & 3,09 & 0,61 & 3,12 \\
\hline
\end{tabular}

Cuadro 5. Resultados para la comparación de b0 y b1 de los modelos por sector mediante la prueba t.

Table 5. Statistical comparison of bo and bl per sector using the test.

\begin{tabular}{|c|c|c|c|c|}
\hline \multirow{3}{*}{$\begin{array}{l}\text { Estimadores de } \\
\text { regresión por } \\
\text { sector }\end{array}$} & \multicolumn{4}{|c|}{ Sectores } \\
\hline & \multicolumn{2}{|c|}{ Horno de Barro } & \multicolumn{2}{|c|}{ Lomas de Toro } \\
\hline & $\mathrm{T}_{\mathrm{m}}$ & $\mathrm{T}_{\mathrm{c}}$ & $\mathrm{T}_{\mathrm{m}}$ & $\mathrm{T}_{\mathrm{c}}$ \\
\hline \multicolumn{5}{|l|}{ Lomas de Toro } \\
\hline b0 (intercepto) & 7,91 & 1,67 & - & - \\
\hline b1 (pendiente) & 3,75 & 1,67 & - & - \\
\hline \multicolumn{5}{|l|}{ Las Animas } \\
\hline b0 (intercepto) & 9,51 & 1,66 & 0,14 & 1,66 \\
\hline b1 (pendiente) & 9,51 & 1,66 & 7,95 & 1,66 \\
\hline
\end{tabular}

2). Posiblemente esto se debió a que el sector Las Animas presentó una mayor apertura del dosel superior que en Horno de Barro debido a la extracción de individuos adultos, tanto de A. chilensis como de robles (Rubilar, 2002). En Horno de Barro existe un dosel más cerrado y una menor alteración antrópica. 


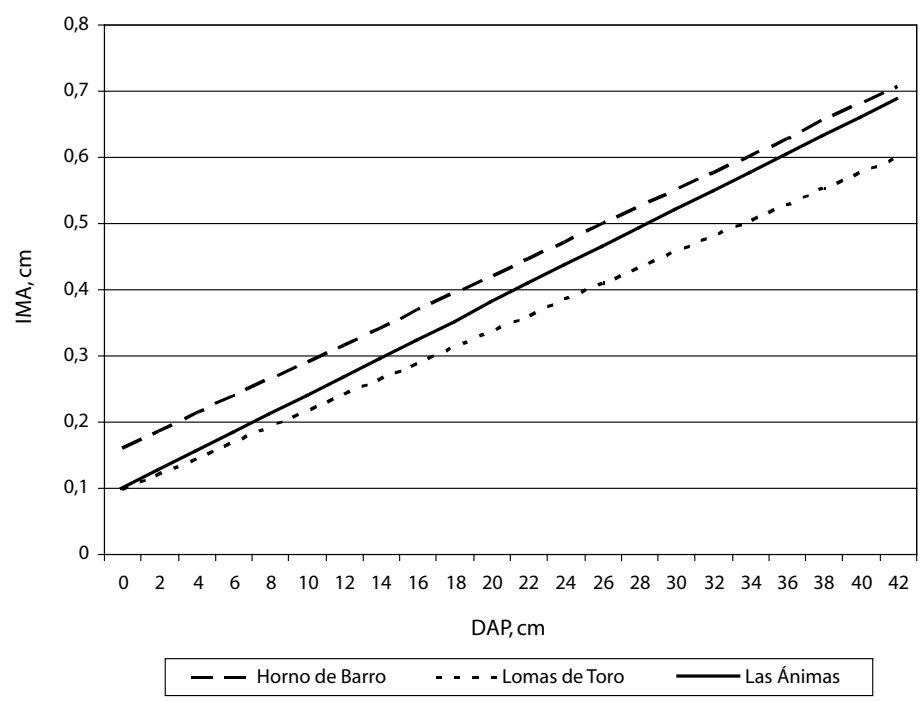

Figura 2. Relación entre el incremento en el diámetro a la altura del pecho (DAP) y el incremento medio anual (IMA) del diámetro de Austrocedrus chilensis obtenido en cada sector muestreado.

Figure 2. Relation between the increment of the diameter determined at chest level (DAP) and the mean annual diameter increment obtained for Austrocedrus chilensis in each study sector.

El sector Lomas de Toro presentó la menor tasa de crecimiento diametral $\left(\beta_{1}=0,012\right)$ (Cuadro $3)$. Esto se debió a que el A. chilensis se expuso a una mayor competencia intraespecífica (Cuadro 2), en suelos pobres y con una mayor densidad de árboles, obtenienda como resultado un crecimiento más regular que en los otros sectores (Tortorelli, 1956). Es así como el desarrollo de los incrementos diamétricos de los árboles de cada sector mostraron dos aspectos relevantes. Por un lado, las característica de individuos que se desarrollaron en condiciones abiertas, representado por Horno de Barro y Las Animas (Cook, 1990); y en contraposición, la diferencia en las pendientes que reflejan distintas situaciones de competencia (Peet y Christensen, 1987), representado por el sector Lomas de Toro, donde se obtuvo una menor tasa de crecimiento. Estas diferencias existentes entre las pendientes de la relación IMA-DAP de cada sector, indicaría que existió un efecto del sitio sobre la tasa de crecimiento del diámetro de los árboles (Dezzotti y Sancholuz, 1991).

\section{Modelo de crecimiento diametral}

Un modelo general de incremento diametral se obtuvo utilizando variables de estado, tanto del árbol sujeto como del competidor, de los tres sectores muestreados. Este modelo permite estimar IMA en DAP de A. chilensis en Maitenrehue, sin diferenciar entre sectores:

$$
\begin{aligned}
& \text { IMA }=\begin{array}{l}
0,127+0,014 \times \text { DAP }-0,008 \times H C C+ \\
0,012 \times \text { DIST COMP }-0,005 \times H_{C C} C
\end{array} \\
& \left(\mathrm{r}=0,88 ; \mathrm{r}^{2}=0,77 ; \mathrm{EEE}=0,07 \mathrm{~cm} ; \mathrm{ECM}=22 \% ;\right. \\
& \mathrm{n}=121)
\end{aligned}
$$

donde IMA $=$ incremento medio anual $(\mathrm{cm})$ en DAP del árbol sujeto, DAP = diámetro $(\mathrm{cm})$ a la altura del pecho (1,3 m) del árbol sujeto, HCC = altura $(\mathrm{cm})$ comienzo de copa viva del árbol sujeto, DIST COMP = distancia (cm) al árbol competidor, $\mathrm{HCC} \mathrm{C}=$ altura $(\mathrm{cm})$ comienzo de copa del árbol competidor.

Al analizar la significación biológica del modelo general construido para IMA, se observó que los mayores coeficientes obtenidos correspondieron a las variables DAP y DIST_COM, con un 0,014 y 0,012 , respectivamente, siendo las variables que mejor explicaron la variación en IMA. A su vez, fueron las variables que presentaron coeficientes positivos (+), indicando una relación directamente proporcional en que a medida 
que aumentó el DAP y la distancia al árbol competidor, aumentó también el IMA.

En contraposición, las variables HCC y HCC_C presentaron coeficientes negativos (-), lo cual indicaría una relación inversamente proporcional; donde a medida que disminuyó la altura de comienzo de copa, tanto del árbol sujeto como del competidor, aumentó el IMA. Esto debido que al existir un aumento en el área foliar hubo mayor capacidad fotosintética, ya que el área foliar se relaciona directamente con la capacidad productiva del árbol (Waring, 1983). Por tal razón, al modelar el IMA con las variables de estado anteriormente mencionadas, se obtuvo que las diferencias de distribución espacial y de tamaño de A. chilensis, determinaron patrones diferentes de crecimiento. Esto se pudo deber a las relaciones de competencia intraespecífica e interespecífica que presenta esta especie, condicionado principalmente por la densidad inicial y la distribución espacial de los individuos (Veblen et al., 1995).

La interacción de todas estas variables señalan que la asociación espacial entre el árbol competidor que acompaña a $A$. chilensis y la altura de la cobertura arbórea, así como los demás patrones de crecimiento diametral mencionados con anterioridad, indican que la tasa de crecimiento de la especie se encuentra supeditada principalmente por la competencia a la cual se encuentre expuesta, reflejando la capacidad de la especie de persistir aún como árbol suprimido (Dezzotti y Sancholuz, 1991). Esto sugiere la existencia de un síndrome de planta nodriza, donde los individuos que crecen en condiciones de alta competencia alcanzan y mantienen por largo tiempo tasas de crecimiento inferiores a plantas que se desarrollan libremente (Veblen et al., 1995). Las variables de estado incluidas en el modelo general logran explicar en un $88 \%$ el incremento medio anual registrado por la especie a distintos niveles de DAP, altura comienzo de copa del árbol sujeto y competidor, además de la distancia existente con el árbol competidor. El modelo presenta un error cuadrático medio de $22 \%$, por lo que la multiplicidad de factores que inciden en el crecimiento pudieron ser explicados con un bajo porcentaje de error (Peri et al., 1996), a través de variables de fácil medición.
En función de los resultados obtenidos se puede concluir que los modelos de incremento medio anual construidos para cada sector, poseen un coeficiente de correlación altamente significativo para las estimaciones de IMA por medio del DAP, constituyendo una base para estimar crecimientos a nivel de árbol individual para cada uno de los sectores muestreados.

Las diferencias existentes entre las pendientes de los modelos de cada sector, nos indican que $A$. chilensis se está desarrollando en calidades de sitio diferentes. El modelo general de incremento medio anual para $A$. chilensis en el sector de Maitenrehue, tiene un coeficiente de correlación de 0,88 , siendo altamente significativo para la estimación de IMA en DAP, al considerar variables de estado pertenecientes al árbol sujeto, árbol competidor y la distancia entre ambos, determinando patrones diferentes de crecimiento, debido a la competencia intraespecífica e interespecífica que presenta la especie. Los modelos ajustados presentaron indicadores de bondad de ajuste con un bajo error, y los múltiples factores que inciden en el crecimiento de A. chilensis pudieron ser explicados a través de variables de fácil medición, brindando una herramienta sencilla para el manejo forestal de la especie.

\section{Resumen}

El estudio se efectuó en tres rodales de "ciprés de la cordillera" (Austrocedrus chilensis) denominados Horno de Barro, Lomas de Toro y Las Animas, en el sector de Maitenrehue (37 $30^{\prime}-39^{\circ} 37^{\prime}$ S; $72^{\circ} 45^{\prime}-73^{\circ} 00^{\prime}$ O), ubicado en la Cordillera de Nahuelbuta, IX Región de la Araucanía, área que es considerada refugio glacial para muchas especies del bosque chileno. Mediante un análisis de árbol individual y la evaluación de su competencia, se construyeron modelos de crecimiento diametral, con la finalidad de explicar y estimar el crecimiento diametral en función de variables dendrométricas y dasométricas de fácil medición. La información se obtuvo mediante análisis de tallo. Como resultado se presentan modelos de incremento medio anual en diámetro a a altura del pecho (DAP) (1,3 m sobre el suelo) para cada sector muestreado, los cuales involucraron como única variable 
predictora el DAP. En segundo lugar, se presenta un modelo general de incremento medio anual para DAP dependiente de la distancia para el sector de Maitenrehue, que involucra variables de estado pertenecientes al árbol sujeto y competidor. Las diferencias estadísticas existentes entre los modelos lineales para cada sector, nos indican que A. chilensis se está desarrollando en calidades de sitio diferentes, y con distintos patrones de crecimiento, debido a la competencia intraespecífica e interespecífica que presenta la especie.

Palabras clave: Ciprés de la cordillera, competencia específica, crecimiento individual.

\section{Literatura citada}

Armesto, J. 1997. Ecología de los Bosques Nativos de Chile. Editorial Universitaria. Santiago-Chile. 477 p.

Baker, F. 1950. Principles of Silviculture. Mc GrawHill Book Company, Inc. NY. USA. 414 p.

CONAF-CONAMA. 1999. Catastro y Evaluación de los Recursos Vegetacionales Nativos de Chile. Informe Nacional con Variables Ambientales. Corporación Nacional Forestal-Corporación Nacional del Medio Ambiente (CONAF-CONAMA). Santiago, Chile. 88 p.

Cook, T. 1990. A guide to using regression equations for estimating tree biomass. North. Journal of Applied Forestry 5:15-22.

Dezzotti, A. y L. Sancholuz. 1991. Los bosques de Austrocedrus chilensis en Argentina: ubicación, estructura y crecimiento. Revista Bosque 12:43-52.

Di Castri, F. y E. Hajek. 1976. Bioclimatología de Chile. Ediciones Universidad Católica de Chile. Santiago, Chile. 129 p.

Donoso, C. 1981. Tipos Forestales de los Bosques Nativos de Chile. Documento de Trabajo No 38 . Investigación y Desarrollo Forestal Corporación Nacional Forestal, Programa de las Naciones Unidas para el Desarrollo-Organización de las Naciones Unidas para la Agricultura y Alimentación (CONAF, PNUD-FAO). Santiago, Chile. $70 \mathrm{p}$.

Donoso, C. 1993. Bosques Templados de Chile y Argentina. Variación, Estructura y Dinámica. Editorial Universitaria. Santiago, Chile. 483 p.

Ferrando, J., J. Goya, M. Barrera, P. Yapura y J. Frangi. 2001. Biomasa y productividad aérea de bosques de Austrocedrus chilensis en Río Negro, Argentina. Revista de la Facultad de Agronomía Universidad Nacional de La Plata (Argentina) 103:139-149.

Goya, J., P. Yapura, J. Ferrando, M. Barrera y M. Arturo. 1998. Crecimiento individual y de rodales de Austrocedrus chilensis en Bolsón, Río Negro, Argentina. Revista de la Facultad de Agronomía Universidad Nacional de La Plata (Argentina) 103:149-155.
Gujarati, D. 1992. Econometría. Mc Graw-Hill. México, D.F. 597 p.

Gutiérrez, L. 2000. Modelación del crecimiento para plantación de Eucalyptus nitens, en suelos precordilleranos andinos de la Provincia del BíoBío, Chile. Seminario de título, Ingeniería de Ejecución Forestal. Universidad de Concepción. Los Angeles, Chile. 22 p.

Martínez, S. 2005. Evaluación de índices de competencia a nivel individual para Pinus radiata (D. DON), en un suelo Precordillerano de la VIII Región. Seminario de título, Ingeniería de Ejecución Forestal. Universidad de Concepción, Los Angeles, Chile. 22 p.

Mora A., Cid L. y M. Valenzuela. 1996. Probabilidades y Estadística. Ediciones Universidad de Concepción. Concepción, Chile. 427 p

Neter, J., M. Kutner, C. Nachtscheim y W. Waserman. 1996. Applied Linear Regression Models. 4th ed. Chicago, Ill: Irwin/McGraw-Hill. Chicago, USA. 720 p.

Peet, R. y N. Christensen. 1987. Competition and tree death. BioScience 37:586-595.

Peri, P. y G. Martínez. 1996. Crecimiento diamétrico de Nothofagus pumilio para dos condiciones copa en un sitio de calidad media de Santa Cruz (Argentina). Investigación Agraria: Sistemas y Recursos Forestales 5:201-212.

Rubilar, H. 2002. Estructura y dinámica de las poblaciones de Austrocedrus chilensis (D. Don) Pic. Serm. et Bizarri, en la Cordillera de la Costa (37³0'S; $72^{\circ} 45^{`}$ W), IX Región de la Araucanía, Chile. Seminario de título, Ingeniería de Ejecución Forestal. Universidad de Concepción. Los Angeles, Chile. 20 p.

Stage, A. 1976. An expression for the effect of aspect, slope and habitat type on tree growth. Forest Science 22:457-460.

Tortorelli, L. 1956. Maderas y Bosques Argentinos. Editorial ACME, S.A.C.I., Buenos Aires. Argentina. $891 \mathrm{p}$.

Trincado, G. y P. Real, 2002. Modelos de crecimiento diametral para Nothofagus obliqua (MIRB.) Oerst y Nothofagus glauca (PHIL.) Kraser. Agro-Ciencia (Chile) 18:53-61.

Veblen, T., B. Burns, T. Kitzberger, A. Lara y R. Villalba. 1995. The Ecology of the conifers of Southern South America. Pages 120-155. In: N. J. Enright and R.S. Hill (eds.). Ecology of the Southern Conifers. Melbourne University Press. Victoria, Australia.

Veit, H. y K. Garleff. 1995. Evolución del paisaje cuaternario y los suelos en Chile central-sur. Páginas 2949. En: J. Armesto, C. Villagrán y M. Kalin Arroyo (eds.). Ecología de los Bosques Nativos de Chile. Editorial Universitaria. Santiago, Chile.

Villagrán, C. 2001. Un modelo de la historia de la vegetación de la Cordillera de la Costa de Chile central-sur: la hipótesis glacial de Darwin. Revista Chilena de Historia Natural 74:793-803.

Waring, R. 1983. Estimating forest growth and efficiency in relation to canopy leaf area. Advance in Ecology Research 13:328-354. 